\title{
Sobre la alienación subjetiva en la organización del trabajo actual. Una observación participante en el comercio de la alimentación al detal
}

\author{
Subjective alienation in current labour organization. A participant \\ observation in a food retail company \\ Héctor L. Bermudez
}

Université du Québec à Montréal (UQAM), Canadá

Recibido el 3 de febrero de 2016; aceptado el 24 de junio de 2016

Disponible en Internet el 18 de noviembre de 2016

\section{Resumen}

El artículo presenta los hallazgos de la primera etapa de un estudio de caso, sobre el placer y el sufrimiento en el trabajo, cuyo trabajo de terreno fue una observación participante en una sucursal de una cadena canadiense de distribución al detal. El propósito es poner a disposición de los estudiosos del análisis organizacional, del management y de la administración de personal algunas definiciones logradas a partir de ciertos recursos bibliográficos (teóricos y metodológicos) de la sociología clínica, la psicodinámica del trabajo y la sociología de las emociones. Se trata de las dimensiones social y técnica de la organización del trabajo y de una forma de alienación subjetiva actual. La principal premisa que puede presentarse, como conclusión, es que la organización actual del trabajo tiende a ser percibida por el trabajador como fuente potencial de amenazas y, sin saberlo, elige protegerse a partir de la alienación subjetiva.

(C) 2016 Universidad Nacional Autónoma de México, Facultad de Contaduría y Administración. Este es un artículo Open Access bajo la licencia CC BY-NC-ND (http://creativecommons.org/licenses/by-nc-nd/4.0/).

Palabras clave: Organización del trabajo; Alienación subjetiva; Sociología clínica; Psicodinámica del trabajo; Sociología de las emociones

Códigos JEL: M12

Correos electrónicos: bermudezhector.1@gmail.com, bermudez.hector_leonel@courrier.uqam.ca

La revisión por pares es responsabilidad de la Universidad Nacional Autónoma de México. 
Abstract

This paper presents the findings of the first stage of a case study of pleasure and suffering at work, whose fieldwork is one participant observation of a Canadian chain of retail. The purpose is to make available to students of organizational analysis, management and human resources, some definitions are finding from some bibliography resources (theoretical and methodological) from clinical sociology, the psychodynamics of work and sociology of emotions. Is about the dimensions social and technical of the work organization and one form of current subjective alienation. The most important premises that can be shows is that the current organization of work tent to be perceives by the employee like a potential threat and, unknowing, they prefers to protect themselves from the subjective alienation.

(C) 2016 Universidad Nacional Autónoma de México, Facultad de Contaduría y Administración. This is an open access article under the CC BY-NC-ND license (http://creativecommons.org/licenses/by-nc-nd/4.0/).

Keywords: Organization of work; Subjective alienation; Clinical sociology; Psychodynamic of work; Sociology of emotions

JEL classification: $\mathrm{M} 12$

\section{Introducción}

En su texto de 1801, Influencia moral de la división del trabajo, Pierre-Edmond Lémontey se preguntaba si la manera particular de organizar el trabajo en su época podría paralizar el pensamiento y la imaginación. Muy al contrario de Adam Smith, Lémontey no encontraba en este tipo de división del trabajo ninguna posibilidad de generar la riqueza de las naciones. Todo lo contrario: el obrero-máquina «hará parte, naturalmente, del pueblo menos dinámico de la Tierra», según Lémontey. Con esta manera de organizar el trabajo podría formarse «una raza de hombres cobarde, degradada, impotente e incapaz de emprender la defensa de la patria» (Lémontey, 1801, p. 7). Aunque más de dos siglos después sus inquietudes parecen vigentes, es necesario insistir en que este tipo de inquietudes no es nuevo en absoluto. Desde los escritos precursores de la economía clásica y de la sociología, por ejemplo, los efectos de la división del trabajo sobre los individuos y sobre la sociedad han sido objeto de investigación. De hecho, en algunos de estos estudios precoces puede constatarse la dramática contradicción del progreso de la división del trabajo en detrimento de ciertas calidades humanas (cf. Comte, 1839; Tocqueville, 1840). Este tipo de paradojas puede también inferirse con toda nitidez, posteriormente, en los estudios fundadores de lo que se conocerá como la Escuela de Relaciones Humanas en su debate con el taylorismo de principios del siglo xx (Mayo, 1933; Barnard, 1938; Roethlisberger y Dickson, 1939), y en los estudios del «Comité para el estudio de las relaciones humanas en la industria», de la Escuela de Chicago (Whyte, 1946, 1949; Gardner y Whyte, 1946).

Sin embargo, el problema nunca ha perdido vigencia académica puesto que, en la realidad empírica, tanto la división social del trabajo como la organización técnica del trabajo se modifican y se ajustan en cada época al mismo tiempo que los efectos sobre las dimensiones sensibles se transforman y se actualizan. Lo anterior, por supuesto, ha exigido a los teóricos reconstruir sus explicaciones. Sin embargo aunque las experiencias del placer y el sufrimiento en el trabajo son manifestaciones evidentes en todo tipo de trabajo, puede constatarse que, aunque históricamente este ha sido un asunto tratado por la teoría organizacional, el management y la gestión de personal, muchas explicaciones al respecto aparecen incompletas y exigen su actualización. En general, estos campos del saber han recurrido, para la explicación de estas experiencias sensibles, 
a ciertos enfoques de la psicología industrial y han privilegiado sus nociones y sus métodos. Sin embargo, el asunto del placer y el sufrimiento en el trabajo cotidiano incluye unas dinámicas que son de una complejidad extrema que escapan a los marcos explicativos más utilizados en estas disciplinas. Por eso, en esta investigación se decidió su examen a partir de marcos teóricos y diseños metodológicos utilizados en trabajos actuales de otros dominios específicos como lo son, por ejemplo, la sociología clínica aplicada al management (De Gaulejac y Hanique, 2015; De Gaulejac, 2005), la sociología de las emociones (Bernard, 2015; Fortino, Jeantet y Tcholakova, 2015), la sociología de la gestión (Linhart, 2015; Salmon, 2015) y la psicodinámica del trabajo (Dejours, 2008; Demaegdt, 2015; Edrei y Gernet, 2015).

Así, el propósito de la investigación que originó el presente artículo es examinar la manifestación de ciertas dimensiones sensibles, como el placer y el sufrimiento cotidiano causados, precisamente, por la organización del trabajo contemporánea. Por tratarse de asuntos inherentes a la naturaleza humana y a sus dinámicas sociales, se decidió privilegiar un enfoque netamente cualitativo. Más adelante, tanto en el apartado del marco teórico como en el de la metodología, se explicará el manejo de ciertos recursos epistemológicos y la utilización de las herramientas de investigación cualitativa; sin embargo, por el momento es necesario mencionar que se diseñó un estudio de caso cuyo trabajo de campo se efectuó a partir de una observación participante, de un semestre de duración, a los jefes inmediatos y su personal subalterno en una sucursal de una gran cadena canadiense del comercio de la alimentación al detal.

Por asuntos de delimitación conceptual (e incluso operatoria) de las categorías centrales de análisis es necesario comenzar con unas definiciones preliminares. Por cuestión de espacio se presenta aquí únicamente la idea central de algunas de estas definiciones. Así, en esta investigación el «placer en el trabajo cotidiano» designa únicamente a los estados episódicos gratificantes llevados a cabo en el ejercicio formal de trabajo en la empresa, en la búsqueda del ser humano «por afirmar y justificar su existencia en la sociedad» (Freud, 1929). En contraste, el «sufrimiento en el trabajo» se define aquí como la experiencia de intensa incomodidad anímica vivida por un sujeto en un momento específico de la realización de sus actividades laborales. En cuanto a las definiciones de «organización del trabajo» y «alienación», estas aparecen aquí mismo en el apartado del marco teórico.

De igual manera, aunque la investigación tiene pretensiones de comprender las dinámicas del placer y el sufrimiento en el trabajo, en tanto que fenómenos universales (es decir, en todo tipo de ejercicio formal de trabajo), ha sido necesario delimitar el estudio a la lógica de la gran corporación privada contemporánea que opera como una «sociedad por acciones» de las que se presentan en los diferentes tipos del capitalismo actual (Amable, 2005). La gran cadena de distribución elegida para el presente estudio de caso es, desde luego, una muestra de este tipo de corporaciones. Por eso, es de esta manera como se comprenderá la noción de «empresa» cada vez que sea enunciada en el presente escrito. En este mismo sentido, es necesario aclarar que por «mandos medios» se comprenderá a todo empleado de la sucursal de tal cadena con responsabilidades de autoridad formal, es decir, el director general, su asistente de dirección, los supervisores, etc. En cuanto al «personal subalterno», este se refiere al que no tiene ninguna autoridad formal: preparadores de pedidos, operadores de mercancías, personal de aseo, etc.

Así, además del problema que se acaba de presentar, el lector encontrará a continuación el marco de referencia que sirve como contextualización histórica y teórica para proponer 3 definiciones originales: a) la dimensión técnica de la organización del trabajo; b) la dimensión social de la organización del trabajo, y c) la alienación subjetiva en el trabajo cotidiano. En segundo lugar, hallará las explicaciones básicas sobre el enfoque metodológico y la estrategia de recolección de la información. En tercer lugar, se verá una discusión sobre algunos resultados que emanaron 
tanto de la revisión de la literatura como del trabajo de campo. El artículo termina con una conclusión general que sintetiza algunos de los hallazgos y propone algunas pistas para futuras investigaciones.

\section{Marco teórico}

\section{La dimensión técnica de la organización del trabajo}

Entre finales de la década de 1970 y comienzos de los años 1980 coincidieron varios fenómenos económicos y sociales que afectaron las maneras taylorista-fordistas de organizar el trabajo y condujeron, en muchos aspectos, a una nueva «lógica organizacional» (Castells, 1998). Entre estos fenómenos destacan, por ejemplo, el agotamiento de la producción de masa, la aplicación de las políticas económicas neoliberales, la crisis de la rentabilidad y de las grandes corporaciones, la invasión de las tecnologías informáticas, etc. La revisión de la literatura muestra que, a partir de los mencionados años 1980, la organización del trabajo en la empresa sufrió, tanto en su dimensión técnica como en la social, una mutación estructural en relación al modelo de organización y de administración anterior ${ }^{1}$. Antes de los años ochenta las empresas se configuraban como estructuras administrativas rigurosamente centralizadas, más o menos autoritarias y verticales, las cuales ya habían sido examinadas tanto por la sociología americana (Selznick, 1948; Gouldner, 1955) como por la francesa (Friedmann, 1946, 1964; Crozier, 1963). Este tipo de estructura organizacional y administrativa fue nombrada por Mintzberg (1979) como «burocracia mecánica». En pocas palabras, se trataba de un modelo de organización en el cual se presenta, a la vez, una relativa claridad en el reparto de las tareas (dimensión técnica), pero también de la distribución de las relaciones de poder (dimensión social). Coriat (1990) calificó tal cambio como la transición del fordismo hacia el post-fordismo, mientras que Castells (1998) recuerda que lo que comenzó en ese entonces fue una transformación radical de la organización del trabajo: la desintegración de la gran firma estructurada según los principios de la integración vertical y de una división (social y técnica) particular del trabajo. La organización vertical fue sustituida por la «firma horizontal», es decir, «una red dinámica y estratégicamente concebida de unidades autoprogramadas y autodirigidas, fundadas sobre la descentralización, la participación y la coordinación» (Castells, 1998, p. 224).

Sin embargo, a pesar de la actualización y la reconfiguración de los modelos de organización del trabajo en la historia reciente, ciertos elementos de la lógica de la separación y de la distribución de las operaciones continúan inamovibles, lo cual permite proponer una definición propia:

En lo que concierne a su dimensión técnica, la organización del trabajo es la separación de todas y cada una de las actividades productivas de la empresa en pequeñas operaciones especializadas para distribuirlas entre los diferentes trabajadores, a manera de tareas, con el fin de hacer el proceso productivo lo más eficaz posible. Esta es concebida y controlada por la alta dirección de la empresa, puesto que emana de la estrategia. Desde este punto de vista, se trata de la lógica del trabajo prescrito, el cual banaliza la experiencia humana en el trabajo (Davezies, 1993). Esto porque la dirección de la empresa tiene necesidad de organizar el trabajo, de ordenarlo de manera racional e interpersonal (Weber) para poder controlarlo, y asume esta dimensión técnica de la organización del trabajo como una causa

\footnotetext{
${ }^{1}$ Las dimensiones «técnica» $\mathrm{y}$ «social» de la división del trabajo han sido categorías bastante estudiadas en ciertas tradiciones de la sociología del trabajo (véase, por ejemplo, Naville, 1961; Gorz, 1973).
} 
para su administración. En otras palabras, la dirección de la empresa tiene necesidad de asumir la organización del trabajo como una condición a fijar, como un dato preexistente.

No obstante, la palabra «organizar» es un verbo, no un sustantivo. Es necesario entonces arriesgar una crítica. Lo que aquí se define como la dimensión técnica de la organización del trabajo no es un dato preexistente. Esta es una de las contribuciones mayores a la psicodinámica del trabajo de parte de los estudios en ergonomía. La relación del ser humano a su trabajo no puede ser estable porque trabajar implica acción; en todas las tareas —incluso en las más simples y divididas - existe una distancia irreducible entre la tarea prescrita y la actividad real del trabajo (Laville, Teiger y Duraffourg, 1973). La tarea prescrita es un ideal, incluso una ilusión. Esta es siempre diseñada en el pensamiento, imaginada primero, para ser elaborada después. Sin embargo, el diseño no garantiza que la tarea sea hecha tal cual que ella ha sido concebida. Cada ejecutor deberá siempre filtrar, en su propia imaginación, su propia inteligencia, etc., la instrucción del diseño y de la concepción de cada tarea. Cada una de ellas deberá pues ser interpretada por el ejecutor. Esto permite asegurar que el trabajo implica un acto de interpretación del mundo de parte de los trabajadores. Pero este acto de interpretación hace que la experiencia real pueda hacerse tan dura, que ocasiona dificultades emocionales que es necesario analizar (Bermúdez, 2013).

En este mismo sentido, la psicodinámica del trabajo (Dejours, 1998), apoyada en lo que se conoce como la «antropología de las técnicas», contribuye a pensar que cuando la dirección de la empresa asume la dimensión técnica de la organización del trabajo como una causa para poderla administrar, no puede ignorar la dialéctica presente en todo ejercicio de trabajo humano: «la técnica es a la vez acto de transformación del mundo y acto de transformación del sujeto» (Dejours, 1998, p. 37), además, los actos técnicos «no tienen la flexibilidad de un proceso simple de interiorización», como el que se describe, por ejemplo, en los manuales de entrenamiento de personal. Lo anterior implica, según este enfoque de la psicodinámica del trabajo, la necesidad de criticar «la noción de "sistema socio-técnico" tan a menudo utilizada en las publicaciones sobre el factor humano» (p. 38). Por eso, se propone aquí invertir la premisa y poner en el centro el examen de las experiencias sensibles de los trabajadores en lugar de presentarlas como consecuencias de un sistema establecido que las determina.

El enfoque «social-construccionista» de la sociología de las emociones, por ejemplo, aunque admite la importancia de los determinantes culturales, procura mantener tal dialéctica en el núcleo de sus desarrollos teóricos. Hochschild (1975) asegura que existe gran plasticidad en las emociones humanas, se aleja de las explicaciones inspiradas en la fisiología y se esfuerza en demostrar la importancia del protagonismo del actor social en el «manejo» de sus emociones: «el individuo efectúa a menudo un trabajo que apunta a producir —o bien a inhibir — sentimientos, al punto de volverlos "apropiados" a la situación» (Hochschild, 2003, p. 19). Por su parte, Shott (1979), inspirada fuertemente en el interaccionismo simbólico, considera que las emociones son experimentadas por el actor social solamente después de que este ha interpretado y definido su situación, y que si bien se trata de «un proceso fuertemente influenciado por definiciones situacionales y normas sociales», las emociones son «una construcción por parte del actor» (Shott, 1979, p. 1318).

\section{La dimensión social de la organización del trabajo}

Los nuevos aspectos de la lógica organizacional de los años 1970/1980 tienen también graves consecuencias sobre la dimensión social de la organización del trabajo. Esta lógica exige unas prácticas de management y de recursos humanos absolutamente diferentes de las anteriores. Así, 
el kanban (o «justo a tiempo»), el Ohnismo (o «cero defectos»), el kaizen (o «mejoramiento continuo»), etc., hacen las delicias de los defensores del management a la americana, el cual inventó una nueva ideología de dirección al apropiarse de las ideas japonesas, las cuales se materializaron en el llamado management «de la excelencia» (Aubert y de Gaulejac, 1991). Sin embargo, lo que resulta sorprendente es que estas nuevas prácticas de dirección desencadenaron una lógica extremadamente conservadora de los aspectos taylorista-fordistas y favorecieron sus efectos perversos sobre las dimensiones humanas: la automatización de los procesos de producción, «a menudo simples modificaciones del trabajo en cadena» (Simonnet, 2009); la división vertical del trabajo como «conservación de las estructuras de dominación» (anunciadas por Weber desde 1921; Weber, 1995); la gestión del control total de la calidad en el proceso de producción (la ilusión del «cero defectos» aparecía ya en Taylor desde 1880$)^{2}$; la incorporación de mecanismos de evaluación individualizada del desempeño (Dejours y Bègue, 2009), etc. Es precisamente la combinación de la nueva manera de organizar el trabajo y de este nuevo tipo de dirección, control y evaluación lo que ha conmocionado la vida cotidiana de las empresas sin, por lo tanto, transformar la lógica de estructuración del poder de la organización del trabajo anterior. Esto permite proponer, entonces, la siguiente definición:

En lo que concierne a la dimensión social de la organización del trabajo, esta es la distribución de todas y cada una de las relaciones (de poder) que los trabajadores establecen entre ellos en el propio escenario del trabajo. De igual manera que en la dimensión técnica, la dimensión social de la organización del trabajo actual está determinada por las decisiones de la alta dirección de la empresa y obedece al diseño de la configuración estructural de cada una de sus divisiones, departamentos, sucursales, etc. Dicho de otra manera, lo que determina esta dimensión social son las relaciones de poder al interior de la empresa, materializada, hoy por hoy, en la exacerbación de la división vertical del trabajo, es decir, el incremento de la separación entre las tareas de concepción y las de ejecución del trabajo.

Es evidente que muchos elementos de la lógica de dominación que subyace a la dimensión social de la organización del trabajo anterior no solamente persisten, sino que se metamorfosean en nuevas figuras, entre las cuales la ya mencionada evaluación individualizada del desempeño, el coaching y otras prácticas comunes del management de «la excelencia», son algunos ejemplos (Londoño y Bermúdez, 2013). En este sentido, la sociología clínica aplicada al management permite examinar la brecha creciente entre los responsables de la concepción de las tareas y los que las ejecutan. De hecho, puede evocarse incluso la paradoja anunciada por Tocqueville desde 1840, con la cual fastidiaba a los defensores de la división del trabajo propuesta por Adam Smith:

Cuando un artesano se entrega sin descanso únicamente a la fabricación de un solo objeto, termina por cumplir con ese trabajo con singular destreza. Pero al mismo tiempo pierde la facultad general de aplicar su espíritu a la dirección del trabajo. Él se vuelve cada día más hábil, pero menos industrioso, y se puede decir que el hombre se degrada, en la medida que el obrero se perfecciona. ¿Qué se puede esperar de un hombre que ha empleado veinte años de su vida a hacer cabezas de alfiler? (Tocqueville, 1840, p. 839).

\footnotetext{
2 Desde la primera página de su publicación Sobre el arte de cortar metales, Taylor (1906) muestra su afán por la calidad del proceso y su tenacidad inquebrantable: «Nuestras investigaciones, que se iniciaron hace 26 años con el propósito definido de encontrar la verdadera respuesta a estas preguntas en todas las condiciones variables de la práctica con las máquinas de los talleres, han sido continuadas hasta hoy día con el mismo objetivo principal todavía a la vista».
} 
La versión actualizada de tal paradoja es denunciada por De Gaulejac (2005, p. 91) cuando explica la relación entre el individuo y lo que él llama la organización managerial del trabajo, es decir, aquella forma de organización del trabajo que emerge del management de la excelencia: «Entre más "exitoso" es el individuo, más aumenta su dependencia. Allí donde la empresa progresa, es el sujeto el que se desmejora. Entre más se identifica a la empresa, más pierde su autonomía». Además, es evidente que las pistas para desentrañar la dimensión social de la organización del trabajo contemporánea, brindadas por la sociología clínica, pueden complementar los enfoques ya mencionados de la sociología de las emociones al aportar para el análisis ciertos recursos explicativos de la teoría psicoanalítica: «En la empresa jerárquica —indica De Gaulejac_ el deseo era reprimido por un Superyó severo y vigilante. En la empresa managerial el deseo es exaltado por un Ideal del Yo exigente y gratificante» (De Gaulejac, 2005, p. 84). En otras palabras, la autorrealización, en el esquema anterior de organización del trabajo, estaba determinada en gran parte por la capacidad de adaptación del trabajador al corpus axiológico de la cultura corporativa, por la sujeción a dicha «cultura», lo cual convertía a la obediencia y la sumisión en valores centrales de este tipo de dominación. Sin embargo, la autorrealización en la nueva organización del trabajo está estipulada por la exhortación al individuo a convertirse en actor de su vida (Martuccelli, 2004, p. 485), lo que invita a pensar en unas nuevas manifestaciones de las lógicas de dominación contemporánea: «las técnicas de management han perdido su carácter disciplinar», ahora de lo que se trata es de «canalizar al máximo la energía libidinal para transformarla en fuerza productiva» (De Gaulejac, 2005, p. 85).

\section{La alienación subjetiva en el trabajo cotidiano}

Diferentes tradiciones intelectuales se han ocupado del concepto de alienación. Además de los fundadores de la sociología (e incluso los precursores), es posible nombrar importantes filósofos: La Boétie, Hobbes, Spinoza, Rousseau, Hegel, Feuerbach, Lukács, Sartre, Althusser y muchos otros $^{3}$. Nisbet (1984) recuerda que varios autores de horizontes tan diferentes como Dostoievski, Burckhardt o Kierkegaard han utilizado en sus escritos elementos estéticos, históricos o filosóficos para dar cuenta de la desintegración social y de la alienación de sus contemporáneos. Sin embargo, en nuestros días la palabra alienación es opaca, entre otras cosas porque ha sido utilizada para definir aspectos muy complejos de dominios completamente diferentes. Así, es posible encontrar algunos desarrollos teóricos tendientes a explicar la alienación en el trabajo (Marx, 1844, 1867; Friedmann, 1946, 1964; Naville, 1957, 1961), la alienación psíquica (Fromm, 1955; Lacan, 1973; Laing, 1969); la alienación política (Long, 1978, 1990); la alienación cívica (Lagrange, 2008; Delaï, 2011), etc.

Por eso, y para centrar la atención en los límites de la presente investigación, será necesario advertir que no se presenta aquí una definición de la alienación en general, sino muy concretamente la de un tipo de alienación subjetiva en el trabajo cotidiano.

Dejours (2006), por ejemplo, se apoya en el esquema desarrollado por Sigaut (2004) desde la antropología de las técnicas. Este último explica 3 formas de alienación: la mental, la social y

\footnotetext{
${ }^{3}$ La palabra «alienación» no es desarrollada en el Discurso sobre la servidumbre voluntaria, pero las ideas de La Boétie en este texto son fundadoras del concepto desde su publicación entre 1546 y 1548, por la influencia que tuvo sobre muchos filósofos, los cuales se encargaron de teorizar la noción (véase: La Boétie, 1546). Igualmente, no aparecen grandes desarrollos ni en Hobbes ni en Spinoza, aunque ambos influenciaron fuertemente a Hegel, sobre todo en lo que concierne al sentido de la «alienación segunda» de los Principios de la filosofía del Derecho (véase Alonso, 1988, pp. 55 y ss.).
} 


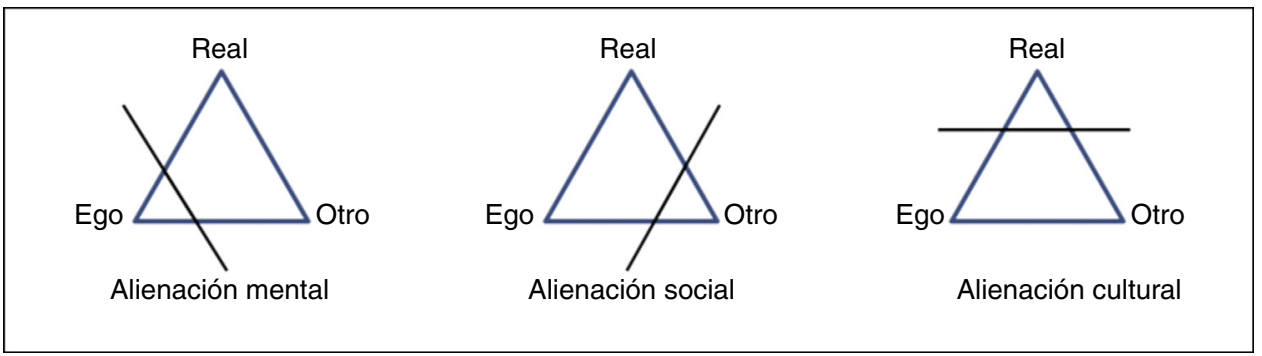

Figura 1. Esquema explicativo de la alienación según François Sigaut. Fuente: Sigaut (2004, p. 121).

la cultural, valiéndose metodológicamente de un triángulo cuyas 3 figuras centrales son el individuo (el Ego), la realidad (lo Real) y los demás (el Otro). Las vinculaciones se establecen por la vía de la solidaridad, el reconocimiento, la identificación. No se trata pues de situaciones o episodios efímeros, sino de identidad, la cual se «construye, deconstruye y reconstruye día tras día». Según Sigaut, la alienación se presenta entonces, cuando se establece una ruptura entre cualquiera de estas vinculaciones (fig. 1).

Este estudio de Sigaut, más la interpretación Dejours, permitieron en nuestro caso proponer la siguiente definición:

La alienación subjetiva en el trabajo cotidiano es la separación que el trabajador establece con respecto de la realidad efectiva, con el fin de defenderse de las amenazas que encuentra en la organización del trabajo. Sin saberlo, en la experiencia real de su trabajo él rompe el vínculo con la realidad (o con el Otro) y le rehúye para protegerse de los peligros que percibe, precisamente, a causa de la doble dimensión (social y técnica) de la organización del trabajo. Así, es posible asociar este tipo de alienación a un mecanismo de defensa que apunta a deshacerse, en el espacio propio de la experiencia de trabajo, de ciertos elementos considerados peligrosos por parte del trabajador. Al experimentar este tipo de vivencia el trabajador pierde su propia capacidad de autorrepresentación y de autonomía, haciéndose incapaz de problematizar muchos aspectos inherentes a la lógica de la organización del trabajo.

En resumen — desde el punto de vista de la dimensión técnica de la organización del trabajo-, la interpretación de la tarea prescrita exige del trabajador un esfuerzo por encontrar el sentido a aquello que él debe hacer, y al mismo tiempo — desde el punto de vista de la dimensión social de la organización del trabajo- la traducción de la instrucción recibida le exige un esfuerzo de aceptación (o de rechazo) del detentor del poder de la que emana. Se asiste entonces a una doble conjunción que amenaza con desestabilizar la sensibilidad del trabajador. Se comprende entonces a la alienación subjetiva como a la separación de un «afuera» que abruma, a la ruptura con un universo que acobarda y que exige protegerse: aquel de la organización del trabajo en la vida cotidiana. Es decir, aparece la alienación como una escapatoria, como un mecanismo de defensa (desesperado, inconsciente, etc., pero siempre justificado). Estas defensas pueden ser individuales (alienación singular), sociales (alienación colectiva) o culturales (alienación masiva). Se decidió entonces llamar alienación subjetiva singular al tipo referido por Sigaut como «alienación mental». Esto para evitar cualquier posibilidad de asociación con la alienación mental descrita por otras disciplinas como la psiquiatría o el psicoanálisis. Se define aquí como la separación del actor social singular, tanto de la realidad establecida como del Otro, entendido como masa. 


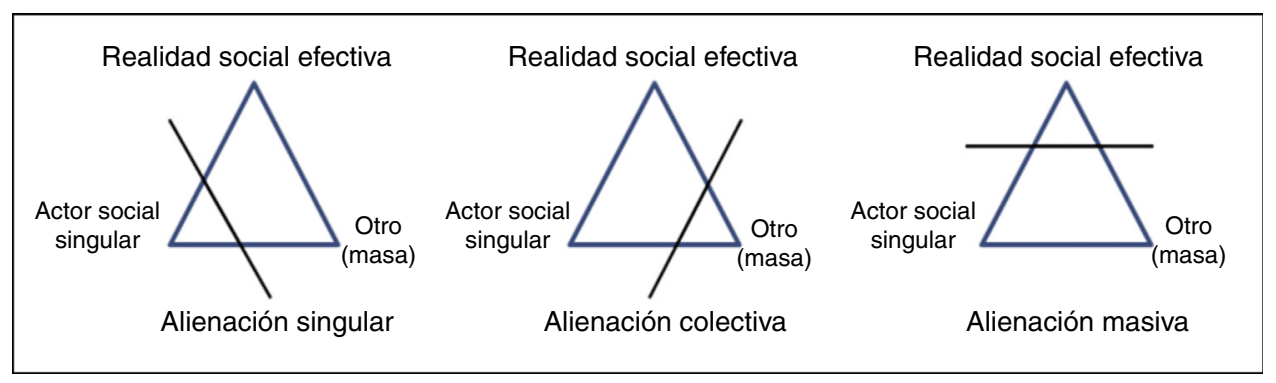

Figura 2. Esquema explicativo de la alienación como ruptura de vinculaciones.

Fuente: adaptado de Sigaut (2004).

En este mismo sentido, en lugar de «alienación social» aquí se prefirió utilizar la categoría de alienación subjetiva colectiva, la cual se define como aquella que se presenta cuando el actor social singular se separa de la masa pero no rompe con la realidad social efectiva, al contrario: le corresponde habitarla, saber bien cómo interpretarla y sin los elementos de protección que le brinda la masa. En tercer lugar, en vez de «alienación cultural» se decidió definir como alienación subjetiva masiva aquella en la cual el actor social singular se refugia en la psicología de la masa (con el Otro) para evadir los dictámenes adversos de la sociedad real efectiva y protegerse del sentimiento de impotencia existencial que le embarga (fig. 2).

\section{Metodología}

Para estudiar un problema como el placer y el sufrimiento cotidiano causados por la organización del trabajo contemporánea, se decidió un enfoque metodológico cualitativo inspirado en los modelos de análisis de la sociología fenomenológica y, en general, en los aportes de la llamada Nueva Escuela de Chicago (Becker, 1963; Garfinkel, 1967; Goffman, 1968; Hughes, 1958; Schütz, 2007). Más concretamente, se llevó a cabo una «observación participante» tal como es definida por Laperrière (1997, p. 242), es decir, «una inmersión total del investigador en la situación social a estudiar» con el fin de lograr «la aprehensión científica de lo real». Se logró pues realizar «un trabajo de análisis intensivo en un escenario restringido» (Quivy y Campenhoudt, 2006, p. 169). Una sucursal del sector de la distribución de alimentos al detal, localizada en Montreal, permitió entonces la observación directa ${ }^{4}$ de las experiencias sensibles en el trabajo cotidiano. Así, el investigador, apoyado por el Departamento de Sociología de la UQAM (Université du Québec à Montréal), consiguió incorporarse a uno de los 3 más grandes detallistas canadienses en alimentación (tabla 1), los cuales, dicho sea de paso, representan más del 50\% de las ventas alimentarias nacionales (Robitaille, Grand, Hitayezu y Kesri, 2015).

Para lograr una «etnología puntillosa» (Castel, 1968) y concentrarse en atrapar las pistas de inteligibilidad que ordenan la coherencia de las experiencias sensibles de los dos grupos

\footnotetext{
${ }^{4}$ Por «observación directa» hay que entender aquí una estrategia metodológica en el sentido ortodoxo de un tipo de observación etnográfica que no privilegia las entrevistas: «Los métodos de observación directa constituyen los únicos métodos de investigación social que captan los comportamientos en el momento mismo en que estos se producen sin la intermediación de un documento o de un testimonio» (Quivy y Campenhoudt, 2006, p. 177). En este mismo sentido, Emerson, Fretz y Shaw, 2010, p. 167) señalan que «la entrevista puede ser útil para obtener precisiones sobre el empleo y la significación de términos y frases particulares, pero la observación por el investigador en situación permite restituir el empleo efectivo y localizado de las categorías indígenas en las interacciones ordinarias».
} 
Tabla 1

Principales cadenas de almacenes de alimentación de Quebec en 2014-2015

\begin{tabular}{|c|c|c|c|c|c|}
\hline \multicolumn{2}{|c|}{ Loblaws-Provigo } & \multicolumn{2}{|c|}{ Sobeys-IGA } & \multicolumn{2}{|l|}{ Metro } \\
\hline $\begin{array}{l}\text { Principales } \\
\text { marcas de tiendas }\end{array}$ & $\begin{array}{l}\text { Número } \\
\text { de tiendas }\end{array}$ & $\begin{array}{l}\text { Principales marcas } \\
\text { de tiendas }\end{array}$ & $\begin{array}{l}\text { Número } \\
\text { de tiendas }\end{array}$ & $\begin{array}{l}\text { Principales marcas de } \\
\text { tiendas }\end{array}$ & $\begin{array}{l}\text { Número } \\
\text { de tiendas }\end{array}$ \\
\hline Provigo & 20 & IGA Extra & 124 & Metro y Metro Plus & 207 \\
\hline Le Marché & 70 & IGA & 158 & Adonis & 6 \\
\hline Provigo Loblaws & 38 & IGA Express & 3 & Super C & 86 \\
\hline $\begin{array}{l}\text { Maxi et Maxi \& } \\
\text { Cie }\end{array}$ & 106 & Marché Tradition & 37 & Les 5 Saisons & 2 \\
\hline Club Entrepôt & 3 & Rachelle Bery & 21 & Dépanneur Gem & 265 \\
\hline Presto & 11 & Marché Bonichoix & 91 & Dépanneur Service & 34 \\
\hline Intermarché & 53 & Bonsoir & 9 & Marché Extra & 199 \\
\hline Axep & 121 & IGA mini & 4 & Marché Ami & 88 \\
\hline & & & & Marché Richelieu & 71 \\
\hline & & & & Dépanneur Serviexp & 2 \\
\hline
\end{tabular}

Fuente: Robitaille et al. (2015).

de empleados elegidos en el estudio de caso, se trabajó durante 5 días por semana cumpliendo horarios de 8 horas por día en los diferentes turnos (en la mañana, en la tarde, en la noche), trabajando incluso los domingos y días feriados. Todo en un período de un semestre. Parafraseando la interpretación que hace Castel (1968) sobre los Asiles de Goffman (1968), puede decirse que se elaboró una «descripción prosaica» de la vida de la mencionada sucursal de la cadena de distribución. Dicha descripción efectuó tal como se observó que la viven los dos grupos de trabajadores elegidos como «población y muestra» de la investigación, es decir, los mandos medios y el personal subalterno.

Se siguió la advertencia de Geertz $(1998$, p. 8) según la cual no hay que volverse indígena para comprender el comportamiento de los nativos que se estudian. Así, en este caso particular el investigador no trató de convertirse en un obrero de la distribución. Más bien este se comprendió como «el observador», en la medida que comprendió a los trabajadores de la sucursal como «los observados». Sin embargo, la experiencia demuestra que, en observación participante, las cosas no son tan simples. Inspirados en Devereux (2012), fue necesario, todo el tiempo, tener en mente que, en ciencias sociales, el carácter unidireccional de la observación es, en gran medida, mera ficción. Esto porque el actor observado tiene, él también, la capacidad potencial de la contraobservación. Lo que se hizo entonces, en el trabajo de campo, fue asumir el rol de trabajador de la cadena de tiendas sin por ello olvidar el rol de científico. Para evitar las perturbaciones a la vida social que se estudió, fue necesario comportarse con los trabajadores observados como un colega, como un compañero; es decir, «camuflarse» en la cotidianidad de la vida de la sucursal para penetrarla: cumplir estrictamente los horarios (registrando su tarjeta de turnos en los relojes de la entrada a la empresa), lucir su misma ropa de trabajo (abrigo, botas, protectores, guantes, insignias); participar de manera rutinaria en las tareas con los trabajadores y ser evaluado por el software que mide el rendimiento individual de cada trabajador de la empresa; impedir cualquier privilegio que la dirección o los representantes sindicales pudieran asignar al investigador, etc. ${ }^{5}$.

\footnotetext{
${ }^{5}$ Resulta útil precisar aquí la estrategia llevada a cabo por el investigador con la que se evitaron las posibles perturbaciones de la vida social de la sucursal en la que se desarrolló la observación participante. En cuanto a la dimensión social de la organización del trabajo se refiere, se procuró, todo el tiempo, acatar las órdenes y poner en práctica un espíritu de
} 
Fue necesario llevar a cabo una toma sistemática de notas a la manera de un diario de bordo (Valéau y Gardody, 2016). Este se elaboró, en la medida de lo posible, de forma discreta para evitar las interferencias en el curso normal de lo cotidiano. Por lo anterior, las notas primarias fueron tomadas en la cafetería, todos los días, antes y después de comenzar las labores. Salvo la curiosidad de algunos trabajadores que se preguntaban qué era lo que un compañero de trabajo escribía diariamente, ninguna perturbación importante fue documentada. El registro de estas observaciones sobre el terreno se realizó en diferentes etapas y niveles, siguiendo las indicaciones de Laperrière (1997, pp. 253-258) y de Valéau y Gardody (2016, p. 80). Es decir, tomando en primer lugar notas estrictamente descriptivas (concretas y neutras sobre lo que se observaba diariamente); luego, haciendo algunos apuntes analíticos (de interpretación de los hechos observados y reflexiones sobre las intuiciones, reacciones del investigador, etc.) y, en paralelo a lo anterior, tomando notas sobre los aspectos metodológicos (la forma de preguntar a los trabajadores sin que se sintieran interrogados, las emociones del investigador como posibles obstáculos a la observación, etc.).

Se evitó llevar a cabo cualquier tipo de entrevista formal (ni siquiera las recomendadas por la ortodoxia de la investigación cualitativa: «abiertas», «no dirigidas», «en profundidad», etc.). Esto, por una parte, para privilegiar la observación directa sobre la indirecta (cf. Emerson, Fretz y Shaw, 2010, p. 167; Quivy y Campenhoudt, 2006, p. 177) e impedir al máximo que los observados se sintieran interrogados. Por otra parte, obedeciendo a una crítica de Howard Becker, según la cual hay que evitar «quedar limitados a lo que se puede recolectar con entrevistas», incluso si estas son de muy larga duración (Becker, 1963, pp. 89-90) ${ }^{6}$.

\section{Resultados y discusión}

La revisión de la literatura y el análisis de la información consignada en el diario de campo permitieron la operacionalización de las principales categorías, tres de las cuales fueron presentadas aquí mismo en el apartado del marco teórico: las dimensiones técnica y social de la organización del trabajo y la alienación subjetiva en el trabajo cotidiano. Entre las preguntas que guiaron el trabajo de campo - preguntas que no se formularon a los trabajadores, sino que orientaron las observaciones del investigador - se incluyeron, por ejemplo, las siguientes: ¿Cómo se expresan las aspiraciones, esperanzas, sentimiento de autoestima y de autorrealización, relaciones de solidaridad y de camaradería, y otras diferentes alegrías en lo cotidiano de sus tareas? ¿Cómo se expresa la experiencia vivida, en lo más profundo de sí, del desgaste anímico causado por un trabajo rutinizado y poco valorado? ¿Cómo se vive el agobio y la degradación de la autoestima, los mutismos, las somatizaciones, los episodios depresivos, etc.? Esto permitió una contrastación entre la teoría sobre las experiencias cotidianas del placer y del sufrimiento generadas por la organización del trabajo, y el examen de las vivencias de los trabajadores que fueron consignadas en el diario de campo.

Se analizaron los hábitos generales, las bromas y tristezas; las armonías y afinidades, las diferencias y altercados; los episodios banales que se mimetizan con las rutinas y también las tragedias o grandes éxitos narrados en el trabajo.

\footnotetext{
sumisión y obediencia semejante al observado en los demás trabajadores en general; en cuanto a la dimensión técnica de la organización del trabajo, se siguieron las instrucciones de las tareas asignadas de acuerdo con las instrucciones y evaluaciones recibidas durante el plan de entrenamiento, también con las descripciones consultadas a los jefes y a los entrenadores y siguiendo los mismos criterios que se observaron en los demás trabajadores.

${ }^{6}$ Becker se refiere aquí al interesante análisis de los problemas metodológicos de las entrevistas «abiertas» [openended] y de larga duración realizado por Neal Gross y Ward S. Mason, quienes examinaron el rol ocupacional de los superintendentes escolares a partir de entrevistas con duración entre 7 y 10 horas (cf. Gross y Mason, 1953).
} 


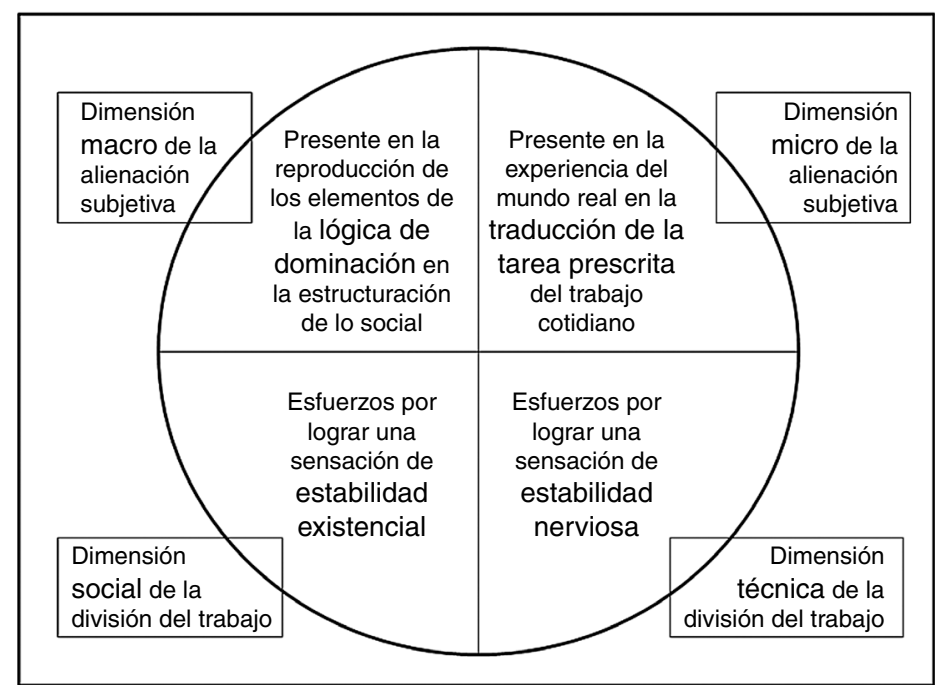

Figura 3. La alienación subjetiva en el trabajo cotidiano.

Fuente: elaboración propia.

Así pudo inferirse que el trabajador se separa, en lugar de apropiarse de la lógica que determina la organización de su trabajo. Esto pudo cotejarse, por ejemplo, con los datos de los autores de la psicodinámica del trabajo (Dejours, 2006, 2004; Edrei y Gernet, 2015), de la sociología clínica aplicada al management (Aubert, 2012, 2003; Enriquez, 2013, 2006; De Gaulejac, 2013, 2011, 2005; De Gaulejac y Hanique, 2015), de la psicología del trabajo (Clot, 2006; Clot, Fernandez y Scheller, 2007), de la ergonomía (Davezies, 2011, 2006, 1993) y de la sociología de las emociones aplicada al trabajo (Soares, 2006, 2003; Fortino et al., 2015).

De esta manera, la definición de las tres mencionadas nociones principales, más otras categorías emergentes del análisis ${ }^{7}$, condujo a la construcción, entre otras hipótesis, de aquella según la cual la dimensión social de la organización del trabajo causa un tipo de alienación subjetiva (macro), la cual está presente en la reproducción de los elementos de la lógica de dominación en la estructuración de lo social (Giddens, 2012; Sales, 2012). En paralelo, la dimensión técnica de la organización del trabajo causaría un tipo de alienación subjetiva (micro) en la experiencia del mundo real en la traducción de la tarea prescrita del trabajo cotidiano (Davezies, 1993; Clot, 2006). Será necesario continuar verificando, en investigaciones futuras, de qué manera los dos ángulos de la alienación se combinan en la lucha de los trabajadores en sus esfuerzos por asegurar una cierta sensación de estabilidad nerviosa y existencial (véase, en la fig. 3, el esquema básico de la hipótesis emergente de esta categorización).

Resulta oportuno continuar preguntándose si el trabajador contemporáneo se aliena en la ejecución de su tarea y obedece a la autoridad de su trabajo para hacer soportable su propia tragedia cotidiana, incapaz de asumirla de manera diferente; seguir investigando si las manifestaciones de placer y la expresión de las experiencias sensibles agradables forman parte del conjunto de elementos con los que cuenta el trabajador para desarrollar y mantener sus

\footnotetext{
${ }^{7}$ Se trata de nociones como la «lógica de dominación», la «tarea prescrita y la tarea realizada», la «estabilidad nerviosa y la existencial», etc. (estas aparecen esquematizadas, aquí mismo, en la figura 3).
} 
niveles de autoestima y autorrealización; indagar si se trata del «trabajo emocional» sobre sus sentimientos, si es acaso la «socialización» de los mismos, según las ideas de Hochschild (2003, p. 19) y de Shott (1979, p. 1319), respectivamente. Sin embargo, puede afirmarse con claridad - porque ha podido comprobarse en el trabajo de terreno- que en la lógica de la organización del trabajo actual en la empresa es realmente todo el universo del trabajo lo que aparece como «dado» al trabajador desde el exterior. La tarea se presenta como una exigencia que él debe, por una parte, interpretar para asegurarse de que está traduciendo bien la realidad; pero, por otra parte, el trabajador debe adaptarse a las instrucciones para evitar el rechazo y el sufrimiento. En otras palabras, la dimensión técnica de la organización del trabajo impide el ejercicio de la autonomía porque la concepción de la tarea aparece proviniendo desde fuera del alcance del trabajador. Al mismo tiempo, la dimensión social de la organización del trabajo impide, igualmente, el ejercicio de la autonomía porque la autoridad de la que emanan las instrucciones está, ella también, por fuera del dominio del ejecutante de la tarea. No obstante, hay que advertir que la mencionada «separación» se presenta como una escapatoria, la cual puede materializarse en la negación de la realidad efectiva; es decir, la «no aceptación» de la experiencia real en el ejercicio del trabajo cotidiano, procurando la invención de un universo menos ingrato.

Precisamente por esto es importante tener en cuenta que, en este mismo contexto, la alienación puede ser asociada también a un mecanismo de resistencia y no solamente a una defensa pasiva. En este sentido, es importante pensar que cuando se percibe una amenaza o un ataque, el mecanismo de defensa puede ser también materializado como una estrategia de resistencia. Es decir, la «escapatoria» no es solamente una huida determinada por la cobardía. La alienación subjetiva en el trabajo sería un mecanismo de protección como aquellos que se encuentran en ciertos ejercicios de resistencia llevados a cabo por los grupos dominados. Al respecto, Scott (2009, p. 154), por ejemplo, recuerda que los grupos vulnerables, más que simplemente «controlar su cólera» en el ejercicio de su resistencia, pueden producir un discurso, el cual (aunque es disimulado con respecto de lo público) procura mantener la dignidad y la afirmación de sí. Igualmente, Burawoy (2008, p. 215), inspirado en Man (1927), considera que, por abrumadora que resulte la tarea rutinaria y «a pesar del embrutecimiento provocado por la mecanización, tiene que existir un margen de iniciativa que permita satisfacer, hasta un cierto punto, el instinto lúdico y la impulsión creativa».

Por eso hoy resulta importante preguntarse por el concepto antagónico al de alienación, como lo es el de «reapropiación», asunto este que inquietó a Naville (1957) en su estudio sobre la «alienación» y «el disfrute» en la obra de Marx y Engels. O por la actualización que de este hace Bernoux (2011) al proponer la «apropiación del trabajo» que llevan a cabo los obreros contemporáneos al modificar las reglas y darle un nuevo sentido a la racionalidad que llevan a cabo en el trabajo cotidiano.

A pesar de que el trabajador percibe la dominación en la organización del trabajo como una fatalidad, no hay una obediencia completa de su parte; no existe una sumisión absoluta de cara a las exigencias de la doble dimensión de la organización del trabajo, porque la tensión en las vinculaciones y las ilusiones necesarias para la dialéctica de la vida social ordinaria no tendrían entonces ningún sentido. Tiene que haber un lugar para las resistencias y para las esperanzas, un sitio para las aspiraciones y para los sentimientos de autoestima y de autorrealización. Así, será necesario continuar interrogándose si la alienación puede ser comprendida como una estrategia sutil de afrontamiento de las situaciones insoportables, como una separación pasajera, de protección, sí, pero también un espacio de relaciones de solidaridad de los trabajadores, de camaradería, para evitar el sufrimiento y la impotencia. 


\section{Conclusiones}

La «alienación» es una categoría filosófica de alcance mayor. Igualmente, la «organización del trabajo» es una noción metateórica del campo de la sociología del trabajo. Por su parte, el «placer» y el «sufrimiento» son conceptos inherentes a la experiencia humana. Para aprovechar la riqueza heurística de este tipo de conceptos y ponerlos al servicio de las explicaciones del análisis organizacional y de la dirección de personal es necesario delimitarlos para lograr convertirlos en categorías operatorias. Esa es una de las principales contribuciones del presente estudio. Para hacerlo fue necesario acudir a campos de estudio diferentes del análisis organizacional, el management y la administración de personal, como lo son la sociología clínica, la sociología de las emociones, la sociología de la gestión y la psicodinámica del trabajo, y cotejar ciertos estudios clásicos de estos campos con sus trabajos más actualizados. En paralelo, se ensayó a comprobar a partir del examen de una porción de la realidad social efectiva — gracias a un estudio de caso cuyo trabajo de terreno fue una observación participante-cómo se presentan las experiencias de placer y sufrimiento en las vivencias de los trabajadores de una gran cadena de distribución alimentaria.

Se descubrió que la organización actual del trabajo puede causar mucha infelicidad, enormes dosis de sufrimiento, la degradación de la autoestima y ciertos estragos sobre la salud física y mental del trabajador contemporáneo. Sin embargo, otra contribución del presente estudio es que es evidente el desequilibrio que existe entre las investigaciones sobre el placer y el sufrimiento en el trabajo cotidiano. En los campos de la sociología y la psicodinámica del trabajo la balanza se inclina fuertemente al considerar — de manera causal y algunas veces simplista— a la organización del trabajo como «culpable» de las miserias del espíritu en las actividades laborales (Dejours, 2015; Edrei y Gernet, 2015; De Gaulejac y Hanique, 2015). En el extremo contrario, la ideología del management y de la administración de los recursos humanos continúa encontrando motivos para negar, ocultar o desconocer la relación entre la organización del trabajo y las desgracias causadas en la salud mental y física del trabajador contemporáneo (Galois-Faurie y Lacroux, 2014; Guillot-Soulez, 2015).

Por eso es importante notar varios hallazgos de la presente investigación. En primer lugar, no todo el mundo sufre en el trabajo; hay quienes afirman ser felices en el desarrollo de sus actividades laborales, y en general se pudo comprobar - tanto en la expresión cotidiana de sus emociones como en sus historias clínicas y ocupacionales- que se mantienen saludables física y mentalmente. En segundo lugar, aunque estudios muy recientes continúan demostrando las «descompensaciones somáticas» severas causadas por la organización actual del trabajo (Edrei y Gernet, 2015), la expresión cotidiana de muchas molestias e inconformidades en el ejercicio de las tareas y rutinas es muestra de un sufrimiento no necesariamente patológico; es decir, no es siempre un sufrimiento invalidante, sino un malestar con el cual puede vivir sin mayores trastornos (Kaës, 1996). En tercer lugar, incluso en los episodios de sufrimiento patológico, los daños causados no son siempre severos y la mayoría de los trabajadores no tienden hacia la depresión y el suicidio. De hecho, aunque los autores especializados en este dominio están de acuerdo en que la causa principal del suicidio por estrés laboral puede hallarse en la organización del trabajo contemporánea, tales casos de suicidio parecen más el síntoma particular de la aplicación de un estilo de dirección en ciertas empresas en coyunturas específicas y resulta bastante riesgoso generalizar las causas de este sufrimiento extremo (Davezies, 2007; Dejours y Bègue, 2009; Du Roy, 2009; Rolo, 2015). Por último, la alienación subjetiva en el trabajo, tal como es definida en el presente estudio, puede considerarse como un poderoso mecanismo de protección que hace que los trabajadores se mantengan, en general, saludables y motivados en sus trabajos. 
Futuras investigaciones deberán encaminarse a continuar estudiando las tensiones y los equilibrios, la búsqueda del placer o la evitación del displacer y todo aquello que hace que, a pesar de las dificultades anímicas y las descompensaciones somáticas causadas por la organización (social y técnica) del trabajo, las personas encuentren y mantengan ciertos grados de felicidad, de alegría y de esperanzas en la vida cotidiana del trabajo formal en la empresa actual.

\section{Referencias}

Alonso, M. (1988). Alienación. Historia de una palabra. México: Universidad Nacional Autónoma de México.

Amable, B. (2005). Les cinq capitalismes. Diversité des systèmes économiques et sociaux dans la mondialisation. París: Seuil.

Aubert, N. (2003). Le culte de l'urgence. París: Flammarion.

Aubert, N. (2012). Le management à l'ère du capitalisme financier: un management hors sujet? Nouvelle Revue de Psychosociologie, 13(1), 17-30. http://dx.doi.org/10.3917/nrp.013.0017

Aubert, N. y de Gaulejac, V. (1991). Le coût de l'excellence. París: Seuil.

Barnard, C. I. (1968). The Functions of the Executive. Cambridge: Harvard University Press (primera edición, 1938).

Becker, H. S. (1963). Outsiders. Studies in the Sociology of Deviance. New York: The Free Press.

Bermúdez, H. L. (2013). Sociología clínica y psicodinámica del trabajo en el estudio del placer y el sufrimiento en el trabajo cotidiano. Teuken-Bidikay, 4, 153-180.

Bernard, J. (2015). Les voies d'approche des émotions. Enjeu de définition et catégorisations. Terrains/Théories, 2 [consultado 25 Jun 2015]. Disponible en: http://teth.revues.org/196

Bernoux, P. (2011). Reconnaissance et appropriation: pour une anthropologie du travail. Esprit, 10, $158-168$. http://dx.doi.org/10.3917/espri.1110.0158

Burawoy, M. (2008). Le procès de production comme jeu. Tracés, 14(1), 197-219. http://dx.doi.org/10.4000/traces.388

Castel, R. (1968). Présentation. En E. Goffman (Ed.), Asiles (pp. 7-35). París: Minuit.

Castells, M. (1998). La société en réseaux. París: Fayard.

Clot, Y. (2006). Clinique du travail et clinique de l'activité. Nouvelle Revue de Psychosociologie, 1(1), $165-177$. http://dx.doi.org/10.3917/nrp.001.0165

Clot, Y., Fernandez, G. y Scheller, L. (2007). Le geste de métier: problèmes de la transmission. Psychologie de l'Interaction, 23/24, 109-138.

Comte, A. (1998). Cours de philosophie positive. Tome quatrième. La philosophie sociale et les conclusions générales. París: Hemann (primera edición, 1839).

Coriat, B. (1990). L'Atelier et le Robot. París: Christian Bourgois.

Crozier, M. (1963). Le phénomène bureaucratique. París: Seuil.

Davezies P. (1993). «Éléments de psychodynamique du travail» [consultado 28 Feb 2012]. Disponible en: http://philippe.davezies.free.fr/download/down/Elements_de_psychodynamique_1993.pdf

Davezies P. (2006). «Activité, subjectivité, santé». [consultado 31 Jul 2013]. Disponible en: http://philippe.davezies.free.fr/download/down/Travail_\%20activite_\%20subjectivite_2006.pdf

Davezies, P. (2007). Suicides. De mauvaises réponses à une vraie question. Santé \& Travail, 60, 29-31.

Davezies P. (2011). «Des origines de la souffrance au travail à sa prévention» [consultado 31 Jul 2013]. Disponible en: http://philippe.davezies.free.fr/download/down/Developpement_prevention_souffrance_au_travail_2011.pdf

De Gaulejac, V. (2005). La société malade de la gestion. París: Seuil.

De Gaulejac, V. (2011). Travail, les raisons de la colère. París: Seuil.

De Gaulejac V. (2013). «Le travail, comme lieu de construction du sujet, entre émancipation et aliénation» [consultado 7 Ene 2013]. Disponible en: http://dc152.4s.io/doc/FpB2rWX0/preview.html

De Gaulejac, V. y Hanique, F. (2015). Le capitalisme paradoxant. Un système qui rend fou. París: Seuil.

Dejours, C. (1998). El factor humano. Buenos Aires: Lumen.

Dejours, C. (2004). La fatigue d'être soi: revers de l'émancipation ou signe d'aliénation? Revue Française de Psychosomatique, numéro spécial «Vivre fatigué», 27-36.

Dejours, C. (2006). Aliénation et clinique du travail. Actuel Marx, 39(1), 123-144. http://dx.doi.org/10.3917/ amx.039.0123

Dejours, C. (2008). Travail, usure mentale. París: Bayard.

Dejours, C. (2015). Pour une clinique de la souffrance au travail. Bulletin de Psychologie, 4(538), $285-291$. http://dx.doi.org/10.3917/bupsy.538.0285 
Dejours, C. y Bègue, F. (2009). Suicide et travail: que faire? París: PUF.

Delaï, S. (2011). Marx, Critique de Feuerbach. París: L'Harmattan.

Demaegdt, C. (2015). Le plaisir au travail et la sublimation à la lumière de la psychodynamique du travail. Le Carnet Psy, 193, 22-26. http://dx.doi.org/10.3917/lcp.193.0022

Devereux, G. (2012). De l'angoisse à la méthode dans les sciences du comportement. París: Flammarion.

Du Roy, I. (2009). Orange stressé. París: La Découverte.

Edrei, B. y Gernet, I. (2015). Le corps entre psychosomatique et psychodynamique du travail: travail de performance et décompensation somatique. Le Carnet PSY, 8(193), 33-38. http://dx.doi.org/10.3917/1cp.193.0033

Emerson, R., Fretz, R. y Shaw, L. (2010). Prendre des notes de terrain. Rendre compte des significations des membres. En D. Cefaï (Ed.), L'engagement ethnographique (pp. 129-168). París: Éditions de l'École des Hautes Etudes en Sciences Sociales.

Enriquez, E. (2006). Violence du pouvoir: la violence subtile dans l'entreprise. Sud/Nord, 1(20), 59-69. http://dx.doi.org/10.3917/sn.020.0059

Enriquez, E. (2013). Le travail, essence de l'homme? Qu'est-ce que le travail? Nouvelle Revue de Psychosociologie, 15(1), 253-272. http://dx.doi.org/10.3917/nrp.015.0253

Fortino, S., Jeantet, A. y Tcholakova, A. (2015). Émotions au travail, travail des émotions. La Nouvelle Revue du Travail, 6 [consultado 16 Feb 2016]. Disponible en: https://nrt.revues.org/2071.

Freud, S. (2007). Le malaise dans la culture. París: PUF (primera edición, 1929).

Friedmann, G. (1946). Problèmes humains du machinisme industriel. París: Gallimard.

Friedmann, G. (1964). Le travail en miettes. París: Gallimard.

Fromm, E. (1955). The Sane Society. New York: Holt.

Gardner, B. B. y Whyte, W. F. (1946). Methods for the study of human relations in industry. American Sociological Review, 11(5), 506-512. Stable URL: http://www.jstor.org/stable/2087256.

Galois-Faurie, I. y Lacroux, A. (2014). Jeux sérieux (serious games) et recrutement: quels enjeux de recherche en gestion des ressources humaines? En P. Paillé (Ed.), Attirer, retenir et fidéliser les ressources humaines. Nouveaux enjeux, nouvelles réponses (pp. 35-60). Québec: Presses de 1’Université Laval.

Garfinkel, H. (2007). Recherches en ethnométhodologie. París: PUF (primera edición, 1967).

Geertz, C. (1998). La description dense. Enquête, 6, 1-20 [consultado 6 Jul 2013]. Disponible en: http://enquete.revues.org/1443.

Giddens, A. (2012). La constitution de la société. Éléments de la théorie de la structuration. París: PUF.

Goffman, E. (1968). Asiles. París: Minuit.

Gorz, A. (1973). Critique de la division du travail. París: Seuil.

Gouldner, A. (1955). Metaphysical Pathos and Theory of Bureaucracy. The American Political Science Review, 49(2), 496-507. http://dx.doi.org/10.2307/1951818

Gross, N. y Mason, W. S. (1953). Some Methodological Problems of Eight-hour Interviews. The American Journal of Sociology, 59(3), 197-204. Stable URL: http://www.jstor.org/stable/2771985.

Guillot-Soulez, C. (2015). La gestion des ressources humaines. Édition 2015-2016. París: Gualino.

Hochschild, A. R. (1975). The Sociology of Feeling and Emotion. En M. Millman y R. M. Kanter (Eds.), Another Voice (pp. 280-307). New York: Doubleday Anchor.

Hochschild, A. R. (2003). Travail émotionnel, règles de sentiments et structure sociale. Travailler, 9(1), 19-49. http://dx.doi.org/10.3917/trav.009.0019

Hughes, E. C. (1958). Men and Their Work. Glencoe: Free Press. http://dx.doi.org/10.3917/trav.009.0019

Kaës, R. (1996). Souffrance et psychopathologie des liens institués. Introduction. En R. Kaës (Ed.), Souffrance et psychopathologie des liens institutionnels (pp. 1-47). París: Dunod.

La Boétie, E. de. (2008). Discours de la servitude volontaire. París: Gallimard (primera edición, 1546-1548).

Lacan, J. (1973). Le Séminaire - Livre XI: Les quatre concepts fondamentaux de la psychanalyse. París: Seuil.

Lagrange, H. (2008). Émeutes, ségrégation urbaine et aliénation politique. Revue Française de Science Politique, 58(3), 377-401. http://dx.doi.org/10.3917/rfsp.583.0377

Laing, R. (1969). La politique de l'expérience. París: Stock.

Laperrière, A. (1997). L'observation directe. En B. Gauthier (Ed.), Recherche sociale: de la problématique à la collecte des données (pp. 241-262). Québec: Presses de l’Université du Québec.

Laville A., Teiger C., Duraffourg J. (1973). «Conséquences du travail répétitif sous cadence sur la santé des travailleurs et les accidents». París: Rapport n. ${ }^{\circ} 29$, Laboratoire de physiologie du travail et d'ergonome, CNAM.

Lémontey, P.-E. (1801). Influence morale de la division du travail. En P.-E. Lémontey (Ed.), Raison, folie, chacun son mot. Petit cours de morale mis à la portée des vieux enfants (pp. 154-180). París: Déterville [consultado 14 Ago 2013 ]. Disponible en: http://www.syllepse.net/syllepse_images/divers/Lemontey.pdf. 
Linhart, D. (2015). Quand l'humanisation du travail rend les salariés malades. Connexions, 1(103), 49-60. http://dx.doi.org/10.3917/cnx.103.0049

Londoño, D. A. y Bermúdez, H. L. (2013). Tres enfoques sobre los estudios críticos del discurso en el examen de la dominación. Palabra Clave, 16(2), 491-519.

Long, S. (1978). Political alienation: Reality and reactance. Journal of Social Psychology, 104, 115-121. http://dx.doi.org/10.1080/00224545.1978.9924043

Long, S. (1990). Explicando la alienación política. Psicología Política, 1, 87-108.

Man, H. (1927). The Psychology of Socialism. New York: Henry Holt.

Martuccelli, D. (2004). Figures de la domination. Revue Française de Sociologie, 45(3), 469-497. http://dx.doi.org/10.3917/rfs.453.0469

Marx, K. (1977). La production de la plus-value relative. En K. Marx (Ed.), Le Capital. Livre premier: Le développement de la production capitaliste (pp. 230-361). París: Éditions Sociales (primera edición, 1867).

Marx, K. (2007). Le travail aliéné et propriété privée. En K. Marx (Ed.), Manuscrits économico-philosophiques de 1844 [Introduit, traduit et annoté par F. Fischbach] (pp. 116-129). París: Vrin (primera edición, 1844).

Mayo, E. (1968). The human problems of an Industrial Civilization. New York: The Viking Press (primera edición, 1933).

Mintzberg, H. (1979). The Structuring of Organizations: A Synthesis of the Research. New Jersey: Prentice-Hall.

Naville, P. (1957). De l'aliénation à la jouissance. La genèse de la sociologie du travail chez. Marx et Engels. París: Marcel Rivière.

Naville, P. (1961). L'automation et le travail humain. París: CNRF.

Nisbet, R. (1984). La tradition sociologique. París: PUF.

Quivy, R. y Campenhoudt, L. (2006). Manuel de recherche en sciences sociales. París: Dunod.

Robitaille, J., Grand, J.-J., Hitayezu, F. y Kesri, K. (2015). Bottin statistique de l'alimentation. Québec: Ministère de l'Agriculture, des Pêcheries et de l'Alimentation du Québec.

Roethlisberger, F. J. y Dickson, W. J. (1976). Management and the Worker. Cambridge, MA: Harvard University Press (primera edición, 1939).

Rolo, D. (2015). Nouvelles problématiques cliniques en psychopathologie du travail: les suicides liés au travail. Le Carnet PSY, 8(193), 39-42. http://dx.doi.org/10.3917/lcp.193.0039

Sales, A. (2012). Sociology Today: Social Transformations in a Globalizing World. London \& Los Angeles: Sage Publications.

Salmon, A. (2015). Néolibéralisme, nouveau management et plaisir. Connexions, 103(1), 21-38. http://dx.doi.org/10.3917/cnx.103.0021

Schütz, A. (2007). Quelques structures du monde-de-la-vie. En A. Schütz (Ed.), Essais sur le monde ordinaire [Préface et traduction de Thierry Blin] (pp. 113-137). París: Le Félin.

Scott, J. C. (2009). La domination et les arts de la résistance. Fragments du discours subalterne. París: Ed. Amsterdam.

Selznick, P. (1948). Foundations of the theory of organization. American Sociological Review, 13(1), 25-35. Stable URL: http://www.jstor.org/stable/2086752.

Shott, S. (1979). Emotion and social life: A symbolic interactionist analysis. American Journal of Sociology, 84(6), 1317-1334.

Sigaut, F. (2004). Folie, réel et technologie. À propos de Philippe Bernardet, Les Dossiers noirs de l'internement psychiatrique, París, Fayard, 1989. Travailler, 2(12), 117-130. http://dx.doi.org/10.3917/trav.012.0117

Simonnet J-P. (2009). «L'organisation du travail industriel: de l'ancienne organisation du travail au taylorisme et au fordisme» [consultado 15 Abr 2013]. Disponible en: http://www.lyc-arsonval-brive.ac-limoges. fr/jp-simonnet/spip.php?article83

Soares, A. (2003). Les émotions dans le travail. Travailler, 9(1), 9-18. http://dx.doi.org/10.3917/trav.009.0009

Soares, A. (2006). Les bleus à l'âme: Le harcèlement psychologique chez les cols bleus de la ville de Montréal. Montréal: UQAM.

Taylor, F. W. (1906). On the Art of Cutting Metals. New York: The American Society of Mechanical Engineers.

Tocqueville, A. de. (2008). De la Démocratie en Amérique (II) París: Flammarion (primera edición, 1840).

Valéau, P. y Gardody, J. (2016). La communication du journal de bord: un complément d'information pour prouver la vraisemblance et la fiabilité des recherches qualitatives. Recherches Qualitatives, 35(1), 76-100.

Weber, M. (1995). Le type pure de domination légale: la direction administrative bureaucratique. En M. Weber (Ed.), Économie et société (pp. 294-301). París: Plon (primera edición, 1921).

Whyte, W. F. (Ed.). (1946). Industry and Society. New York: McGraw-Hill.

Whyte, W. F. (1949). The Social Structure of the Restaurant. American Journal of Sociology, 54(4), 302-310. Stable URL: http://www.jstor.org/stable/2770650 